\title{
The role of the concept of biologically effective dose (BED) in treatment planning in radiosurgery
}

\author{
William T. Millar a, John W. Hopewell ${ }^{\text {b, * }}$, Ian Paddick ${ }^{c}$, Christer Lindquist $^{\text {c }}$, \\ Håkan Nordströn ${ }^{\mathrm{d}}$, Pär Lidberg ${ }^{\mathrm{d}}$, Jonas Gårding ${ }^{\mathrm{d}}$ \\ ${ }^{a}$ Beatson Oncology Centre, Gartnavel Hospital, Glasgow, UK \\ b Green Templeton College and Particle Therapy Cancer Research Institute, University of Oxford, Oxford, UK \\ ${ }^{\mathrm{c}}$ Cromwell Hospital, Cromwell Road, London, UK \\ d Department of Research and Development, Elekta Instrument, Stockholm, Sweden
}

\section{A R T I C L E I N F O}

\section{Article history:}

Received 20 December 2014

Received in revised form

12 April 2015

Accepted 13 April 2015

Available online 14 May 2015

\section{Keywords:}

Radiosurgery

Treatment planning

Biological effective dose

\begin{abstract}
A B S T R A C T
Radiosurgery (RS) treatment times vary, even for the same prescription dose, due to variations in the collimator size, the number of iso-centres/beams/arcs used and the time gap between each of these exposures. The biologically effective dose (BED) concept, incorporating fast and slow components of repair, was used to show the likely influence of these variables for Gamma Knife patients with Vestibular Schwannomas. Two patients plans were selected, treated with the Model B Gamma Knife, these representing the widest range of treatment variables; iso-centre numbers 3 and 13, overall treatment times 25.4 and $129.6 \mathrm{~min}$, prescription dose $14 \mathrm{~Gy}$. These were compared with 3 cases treated with the Perfexion ${ }^{\circledR}$ Gamma Knife. The iso-centre number varied between 11 and 18, treatment time $35.7-74.4$ min, prescription dose $13 \mathrm{~Gy}$. In the longer Model B Gamma Knife treatment plan the $14 \mathrm{~Gy}$ iso-dose was best matched by the $58 \mathrm{~Gy}_{2.47}$ iso-BED line, although higher and lower BED values were associated with regions on the prescription iso-dose. The equivalent value for the shorter treatment was $85 \mathrm{~Gy}_{2.47}$. $\mathrm{BED}$ volume histograms showed that a BED of $85 \mathrm{~Gy}_{2.47}$ only covered $\sim 65 \%$ of the target in the plan with the longer overall treatment time. The corresponding BED values for the 3 cases, treated with the Perfexion ${ }^{\circledR}$ Gamma Knife, were 59.5, 68.5 and $71.5 \mathrm{~Gy}_{2.47}$

In conclusion BED calculations, taking account of the repair of sublethal damage, may indicate the importance of reporting overall time to reflect the biological effectiveness of the total physical dose applied.
\end{abstract}

(C) 2015 Published by Elsevier Ltd on behalf of Associazione Italiana di Fisica Medica. This is an open access article under the CC BY-NC-ND license (http://creativecommons.org/licenses/by-nc-nd/4.0/).

\section{Introduction}

In the current clinical practice of radiosurgery (RS), treatment is usually defined by the total physical dose to an iso-surface which is conformed as close as is practically possible to the gross tumour volume (GTV). By way of an example, using the Gamma Knife, a standard approach is to specify the total physical treatment dose prescribed to the $50 \%$ iso-dose, with no margin. The prescription dose is thus the maximum dose to normal tissue and the minimum dose to tumour. In this instance the peak dose to the tumour will be twice the prescription dose as reflected in dose volume histograms

\footnotetext{
* Corresponding author. Green Templeton College, University of Oxford, Oxford, UK.

E-mail address: john.hopewell@gtc.ox.ac.uk (J.W. Hopewell).
}

(DVH). With the introduction of dose optimizing approaches to maximize the conformity of the prescription iso-dose to the GTV and to maximize the dose gradient outside the target, the prescription iso-dose can vary $[1,2]$. Consequently the maximum dose will differ for a given prescription dose, even though the gradient at the target periphery may be more consistent between different treatment plans.

The prescribed total physical dose in a single treatment session in RS has historically been referred to as a single dose exposure. However, as pointed out previously, this is not the case for treatments involving multiple iso-centres using the Gamma Knife. The dose is actually delivered in multiple exposures with varying time gaps $[3,4]$. This, to a greater or lesser extent, also applies to other RS technologies. Using the Gamma Knife the number of iso-centres used reflects the complexity of the plan required to cover the lesion, although this is subject to practical constraints when 
collimators are changed manually. With the advent of more automated technology, (Perfexion ${ }^{\circledR}$ ), the number of iso-centres used tends to increase for comparable lesions in order to maximise conformity, which can affect overall treatment times. However, the time-related decay in the activity of the Cobalt- 60 sources will also have a potentially significant effect on overall treatment time for a given treatment. The overall treatment time should include any gaps in treatment and not just the radiation beam-on time [3,4]. A single iso-centre represents the focus of 201 individual collimated Cobalt-60 sources in the case of the Model B Gamma Knife and 192 sources in the case of Perfexion ${ }^{\circledR}$, although individual, or groups of collimators (sectors) may be blocked to reduce the doses to critical structures.

Currently these variations in the overall treatment time are not taken into account in treatment planning. However, it is well known even from early cell survival studies that protraction of a given dose, by using lower dose-rates, will lead to a loss of biological effectiveness [5]. Concerns were also expressed following the introduction of Intensity Modulated Radiotherapy (IMRT) when conventional $2 \mathrm{~Gy}$ fractions were extended beyond the few minutes used in conventional radiotherapy. A dose of 2 Gy gradually lost efficacy, in terms of an increase in clonogenic cell survival, as the exposure time was gradually increased from a few minutes up to $1 \mathrm{~h}$ [6]. Likewise, in terms of normal tissue toxicity, protraction of the dose has been found to correlate with a decrease in toxicity. For example, in studies involving the uniform irradiation of a $10 \mathrm{~cm}$ length of spinal cord in pigs, a single dose of 25 Gy delivered in $\sim 30$ min resulted in a $100 \%$ incidence of radiation myelopathy; that same dose delivered in $\sim 140$ min resulted in no cases of myelopathy (Hopewell, unpublished data).

In addition, within a given patient, there are regional variations in the constituent doses that are summed, from the different isocentres, to produce a given total physical dose. For example for an individual voxel on a given physical dose iso-surface there will be variation in the dose-rate due to the collimator used and geometric orientation for the different iso-centres used. These factors have implications for the biological effectiveness of the total physical dose prescribed to that voxel. For some voxels, the majority of the total dose delivered will come from a single iso-centre, with relatively little contribution from any other iso-centres used in the treatment. At another anatomical location, on the same total physical iso-surface, the relative dose contribution from the different iso-centres may be much more uniform. This variation in the actual dose protocol delivered to different voxels on the same total physical iso-surface impacts upon the biological effectiveness of a given total radiation dose since in the linear quadratic (LQ) model, biological effectiveness is related to the sum of the dose from each iso-centre and the dose squared (moderated by the doserate considerations), as illustrated previously in relation to RS [4]. Voxels with the highest dose contribution from a single iso-centre will have the highest biological effective doses (BED). On a single physical iso-dose surface, the biological effective dose may vary by $\sim 15 \%$ in normal tissue for a given physical dose based treatment plan [4].

In this paper the role of the concept of biologically effective dose on treatment planning in RS is explored since at present the wide variation between different treatment plans does not make it intrinsically obvious as to the likely biological effectiveness of any prescription dose. This is particularly so for different Models of Gamma Knife where the contribution of gaps in exposure to the overall treatment time vary widely, e.g. with the Model B Gamma Knife time gaps contribute approximately $50 \%$ to the overall treatment time, while with Perfexion ${ }^{\circledR}$ it is only 2-3\%. Two Model B treatment plans for Vestibular Schwannomas cases, representing each end of the spectrum in terms of complexity and treatment duration, both treated with a physical prescription dose of $14 \mathrm{~Gy}$ to the $50 \%$ iso-dose, were chosen. These were compared with three individual treatments using the Perfexion ${ }^{\circledR}$ Gamma Knife. The latter patients received a prescription dose of $13 \mathrm{~Gy}$, to slightly different iso-dose levels, but with overall treatment times that were in the same range associated with the treatment of the first two patients. Physical treatment plans, and their associated BED plans, were compared on the basis of iso-dose/iso-BED surfaces on MRI images and from the comparison of DVH and BED volume histograms (BEDVH). The objective was to obtain a better understanding of the range of BED values and the factors influencing them in order to allow the design of an appropriate retrospective study that would relate BED values to tumour response/normal tissue complication probability, irrespective of the design of Gamma Knife or other form of RS that might be used.

\section{Materials and methods}

\section{Patient population}

Two cases were selected from a group of 26 patients treated for a Vestibular Schwannoma with a Model B Gamma Knife that were included in an earlier study [4]. Both were treated with a prescription dose of $14 \mathrm{~Gy}$ to the $50 \%$ physical iso-dose. The protocol for the first patient involved the use of 3 iso-centres, delivered in an overall treatment time of $25.4 \mathrm{~min}$ ( 2 gaps each of $6 \mathrm{~min}, 13.4 \mathrm{~min}$ of beam-on time), the second with a total of 13 iso-centres (12 gaps each $6 \mathrm{~min}, 57.6 \mathrm{~min}$ of beam-on time) in an overall treatment time of $129.6 \mathrm{~min}$.

The results from the above 2 cases were compared with three patients treated for Vestibular Schwannomas, with a prescription iso-dose of $13 \mathrm{~Gy}$ using a newer version of Gamma Knife, Perfexion $^{\circledR}$. These treatments represent the maximum range of overall treatment times that have been used with these two types of Gamma Knife at the Cromwell Gamma Knife Centre, London. The characteristics of these cases are listed in Table 1. The two physical doses were the most routinely used for patients treated using the Model B and Perfexion ${ }^{\circledR}$ Gamma Knife, respectively.

\section{Calculation of regional variation in biological effective dose}

In order to calculate the dose prescription to various points in the region of interest a prototype research version of Leksell GammaPlan ${ }^{\circledR}$ has been developed as described previously [4]. Briefly, in this version of Leksell GammaPlan ${ }^{\circledR}$, the dose-rate associated with each iso-centre used could be extracted for each voxel in a $31 \times 31 \times 31$ matrix covering the selected region of interest. Since the time of exposure for each iso-centre is known the dose contribution from each iso-centre in each voxel could subsequently be calculated. It is the summation of the physical dose from each iso-centre that provides the total physical dose to a specific voxel. The BED for each voxel in the $31 \times 31 \times 31$ matrix can be calculated using the following equation:

$B E D=\mathrm{D}_{\mathrm{T}}+\frac{1}{\alpha / \beta}\left[\frac{\phi\left(\Xi, \mu_{1}\right)+\mathrm{c} \phi\left(\Xi, \mu_{2}\right)}{1+\mathrm{c}}\right] \sum_{\mathrm{i}=1}^{\mathrm{n}} \mathrm{d}_{\mathrm{i}}^{2}$

where $\phi(\Xi, \mu)$ is a complicated function of the protocol and the repair rates see ref [7]; the effects of dose-rate, the inter-iso-centre time and the exposure time are mediated via this function. ' $\mu_{1}$ ' and ' $\mu_{2}$ ' represent two sublethal radiation damage repair rates that are associated with protracted exposures and ' $c$ ' the partition coefficient of the slower component (' $\mu_{1}$ ' $>$ ' $\mu_{2}$ '). It should be noted that the absolute partitioning between the two repair processes ' $\mu_{1}$ ' and 
Table 1

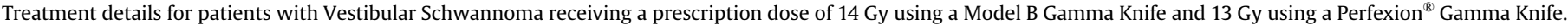

\begin{tabular}{|c|c|c|c|c|c|c|}
\hline Patient no & Gamma knife type & Tumour volume (cc) & Prescription iso-dose (\%) & Number of iso-centres & Beam on time (min) & Overall time $(\mathrm{min})^{\mathrm{a}}$ \\
\hline 1 & Model B & 2.3 & 50 & 3 & 13.4 & 25.4 \\
\hline 2 & & 6.0 & 50 & 13 & 57.6 & 129.6 \\
\hline 1 & Perfexion $^{\circledR}$ & 4.81 & 50 & 13 & 34.5 & 35.7 \\
\hline 2 & & 1.97 & 47 & 11 & 46.3 & 47.3 \\
\hline 3 & & 2.42 & 52 & 18 & 72.7 & 74.4 \\
\hline
\end{tabular}

a Average interval of 6.0 or 0.1 min assumed between each iso-centre is included in the overall treatment time for Series B and Perfexion ${ }^{\circledR}$ Gamma Knives, respectively.

' $\mu_{2}$ ' is $1.0 /[1+c]$ and $c /[1+c]$, respectively. The total dose, ' $D_{T}$ ', is the summation of the different doses delivered to a given voxel by the treatment, delivered by the ' $n$ ' iso-centres, where ' $d_{i}$ ' $(i=1, n)$ is the dose delivered by each of the ' $n$ ' different iso-centres. Details of the derivation of this model and the definition of $\phi(\Xi, \mu)$ can be found elsewhere [7-9]. Experiments in the rat spinal cord, using single dose exposures delivered at different dose-rates were used to derive the values of the repair kinetic parameters for normal CNS tissue that have been used in this analysis [10]. Half-times for the repair of sublethal irradiation damage of $0.19 \mathrm{~h}\left(\ln 2 / \mu_{1}\right)$ and $2.16 \mathrm{~h}$ $\left(\ln 2 / \mu_{2}\right)$; partition coefficient ' $c$ ' $(0.98)$, were obtained from these studies with an associated $\alpha / \beta$ ratio of $2.47 \mathrm{~Gy}$. Comparable $\alpha / \beta$ ratios and half-times for repair have been derived from studies using more conventional fractionated irradiation, with either complete or incomplete inter-fraction repair intervals, or low and very low dose-rates have been discussed in detail elsewhere $[4,11]$. It should be specifically noted that these repair processes are deemed to act concurrently resulting in a composite tissue response. These values have been adopted for use in the present study to calculate the BED to the normal brain stem; the tumours treated causing some compression of this normal tissue structure. Comparable radiobiological parameters are not available for Vestibular Schwannomas, but in this slowly growing, well differentiated, tumour it would not be unreasonable to suggest that they may be similar to those for normal CNS tissue.

The $31 \times 31 \times 31$ matrix of total physical dose values and BED values were used to construct relative volume histograms i.e. DVHs and BEDVHs. For the DVHs the volume was normalized to the appropriate physical prescription dose, while for the BEDVHs only the BED values for those voxels that had total physical doses of $\geq 14 \mathrm{~Gy}$ or $\geq 13 \mathrm{~Gy}$ were used to construct the BEDVH for Model B and Perfexion ${ }^{\circledR}$ cases, respectively. The matrix of BED values was also reimported back into the prototype research version of Leksell GammaPlan ${ }^{\circledR}$ so that BED iso-lines could be compared with physical iso-dose lines on the same MR images.

\section{Results}

Studies of cases treated using the model B gamma knife

The frequency distribution of BED values for voxels irradiated within the $31 \times 31 \times 31$ matrix that received total physical doses of 14,13 and $12 \mathrm{~Gy}$, are presented in Fig. 1 for the patient treated with 13 iso-centres (129.6 min). The tumour volume was $6.0 \mathrm{cc}$. In order to obtain this BED value distribution the physical dose range evaluated was widened slightly $( \pm 0.02 \mathrm{~Gy})$ to increase the number of voxels sampled. BED values for each physical dose were then placed in bins, e.g. 50.00-50.99 Gy; 51.00-51.99 Gy etc. The peak frequencies of BED values obtained were $45.5,52.5$ and $58.0 \mathrm{~Gy}_{2.47}$, for total physical iso-doses of 12 (green), 13 (red) and 14 Gy (blue), respectively. In the subsequent discussion, BED values derived in this way will be defined as the 'most representative' of a given total physical dose. For simplicity only the corresponding 'most representative' BED values for the 3 iso-centre (25.4 min) treatment are given in Fig. 1. The range of BED values were 60.3-66.21, $68.37-76.49$ and $76.74-85.29 \mathrm{~Gy}_{2.47}$ for the total physical doses 12 , 13 and $14 \mathrm{~Gy}$, respectively. Although these two patients were prescribed the same total physical dose of $14 \mathrm{~Gy}$ to the $50 \%$ iso-dose, the impact of the treatment delivery on the biological effectiveness of that dose was such that the patient who had the 13 isocentre treatment only received a dose equivalent to $\sim 12 \mathrm{~Gy}$, when compared with the other patient.

The difference between these two treatments is further exemplified by a comparison of their BEDVHs relative to the conventional DVHs (Fig. 2). The conventional DVHs for the two patients are similar for total doses between 14 and $18 \mathrm{~Gy}$ (relative volume 100-55\%), then diverging slightly, converging at the upper dose limit of $28 \mathrm{~Gy}$. Given that the BED value most representative of the 3 iso-centre treatment was $85 \mathrm{~Gy}_{2.47}$ with an upper value of $270 \mathrm{~Gy}_{2.47}$ representing $28 \mathrm{~Gy}$, for total physical doses of 14 and $28 \mathrm{~Gy}$, the BED axis has been scaled accordingly. Based on this scaling the BEDVH for the 3 iso-centre patient is reasonably consistent with the DVH. However, the BEDVH for the 13 iso-centre patient is markedly shifted to the left. The proportion of the $14 \mathrm{~Gy}$ volume covered by a BED of $85 \mathrm{~Gy}_{2.47}$ in the patient is only $\sim 65 \%$ (see Fig. 2). This difference in coverage can further be illustrated by the comparison of total dose and BED iso-lines on a treatment plan image for each of the two treatments (Fig. 3).

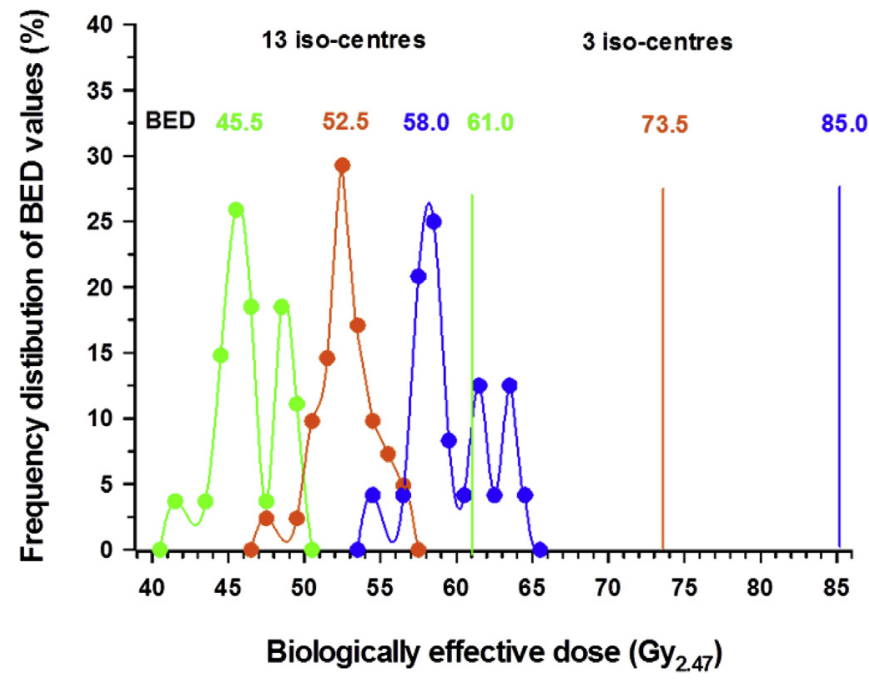

Figure 1. Frequency distribution of BED values in a patient treated using 13 isocentres, prescription dose $14 \mathrm{~Gy}( \pm 0.02 \mathrm{~Gy})$ with a Model B Gamma Knife (blue). The BED values for that iso-dose are compared with those for 13 (red) and $12 \mathrm{~Gy}$ $( \pm 0.02 \mathrm{~Gy})$ (green). The comparable most representative BED values for a 3 iso-centre treatment, prescribed $14 \mathrm{~Gy}$ are also plotted for comparison. (For interpretation of the references to colour in this figure caption, the reader is referred to the web version of this article.) 


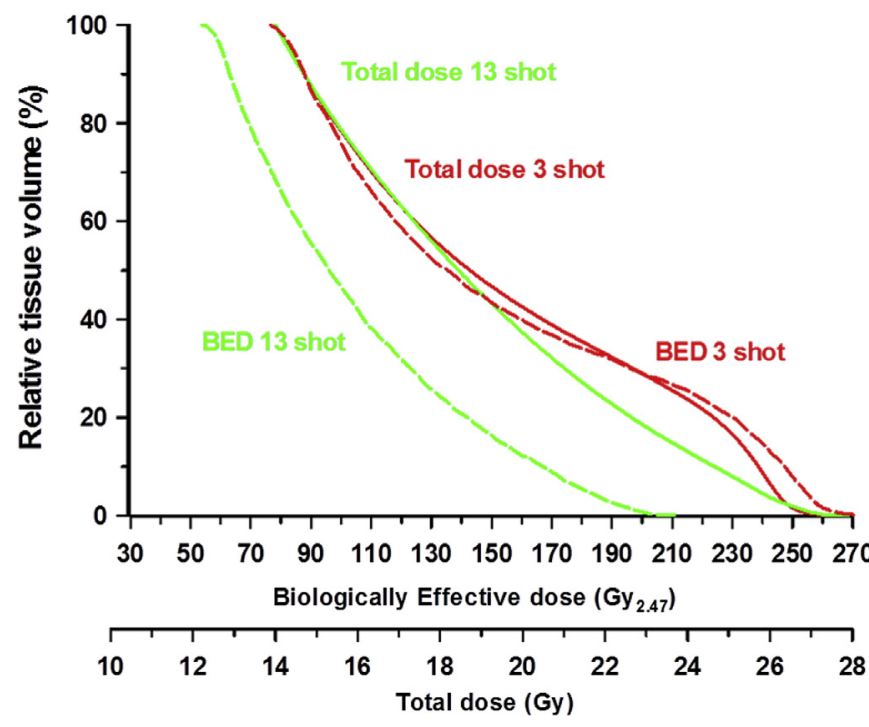

Figure 2. Dose volume histograms (solid lines) for the two patients treated with a model B Gamma Knife illustrated in Figure 1 (prescription dose 14 Gy - maximum dose $28 \mathrm{~Gy}$ ). BED volume histograms, for the same two patients, are given for comparison (broken lines). A BED value of $85 \mathrm{~Gy}_{2.47}$, that was most representative of the prescription dose of $14 \mathrm{~Gy}$ in the patient treated with 3 iso-centres, only covered $\sim 65 \%$ of the lesion in the patient treated with 13 iso-centres.

\section{Studies of cases treated using the Perfexion ${ }^{\circledR}$ gamma knife}

The BED values most representative of the prescription dose of $13 \mathrm{~Gy}$ and the lower iso-dose of $12 \mathrm{~Gy}$, in the three Perfexion ${ }^{\circledR}$ patients evaluated (summarized in Table 1), are given in Table 2, along with the range of BED associated with these physical doses. In these treatments the prescription iso-dose varied above and below the $50 \%$ iso-dose and thus the peak physical dose in the lesions varied between 25 and $27.8 \mathrm{~Gy}$. The resulting DVHs although, by definition, co-incident at the prescription dose of $13 \mathrm{~Gy}$, varied at higher doses due to differences in the internal dose gradient between the plans, a function of the iso-centre configuration and prescription iso-dose selected (Fig. 4). The BEDVHs for the same three Perfexion ${ }^{\circledR}$ cases are also plotted and compared with the
BEDVHs for the two Model B patients. The BEDVH for the Perfex$\operatorname{ion}^{\circledR}$ case treated with a prescription dose of $13 \mathrm{~Gy}$ in the longest overall treatment time (69.1 min) was very comparable with that for the model B cases treated in the longest overall treatment time (14 Gy - $129.6 \mathrm{~min}$ ). The BEDVH for the Perfexion ${ }^{\circledR}$ case treated with the shortest overall treatment time was similar to that for the model B case treated in 25.4 min with $14 \mathrm{~Gy}$. In all three Perfexion ${ }^{\mathbb{R}}$ cases the $\geq 85 \mathrm{~Gy}_{2.47}$ BED coverage of the target was less than $100 \%$, being $~ 60 \%$ in the cases treated in the longest overall treatment time. For the prescription dose in these cases, at the junction between normal brain stem and tumour, there is little difference in the range of BED values between a physical iso-dose of $13 \mathrm{~Gy}$ delivered by Perfexion ${ }^{\circledR}$ and 14 Gy delivered by the Model B Gamma Knife.

\section{Discussion}

In RS treatments using the Gamma Knife, the beam-on-time that is required to deliver a given prescription dose varies widely. Prolongation predominantly relates to the complexity of the treatment plan, largely dependent on an increase in the number of iso-centres used, on sector/beam blocking, as well as on the activity of the cobalt-60 sources at the time of treatment. When the time interval between iso-centres is added to the beam time, to provide the overall treatment time, then this will be greatly influenced by the gap between the individual iso-centres for the older Model B Gamma Knife. For example, in the present study, set up time was approximately $47 \%$ and $55.5 \%$ of the total of $25.4 \mathrm{~min}$ and $129.6 \mathrm{~min}$, respectively, for the two cases assessed. This assumes an average interval of 6 min between dose delivery, from each of the isocentres. With the introduction of the Perfexion ${ }^{\circledR}$ Gamma Knife the contribution of gaps to the overall treatment time is significantly reduced, being in the order of $2-3 \%$ of the overall treatment time in the 3 cases selected for the present analysis. However, there was still a wide spread in the overall treatment time for the same physical prescription dose in these patients. Despite these major differences in overall treatment time, no account is currently taken of this in treatment planning in order to adjust for any change in the biological effectiveness of a given radiation dose. This implies that there is a belief that there is no repair of sublethal radiation damage associated with the variations in overall treatment times associated with RS treatments. Such a conclusion is not consistent with the

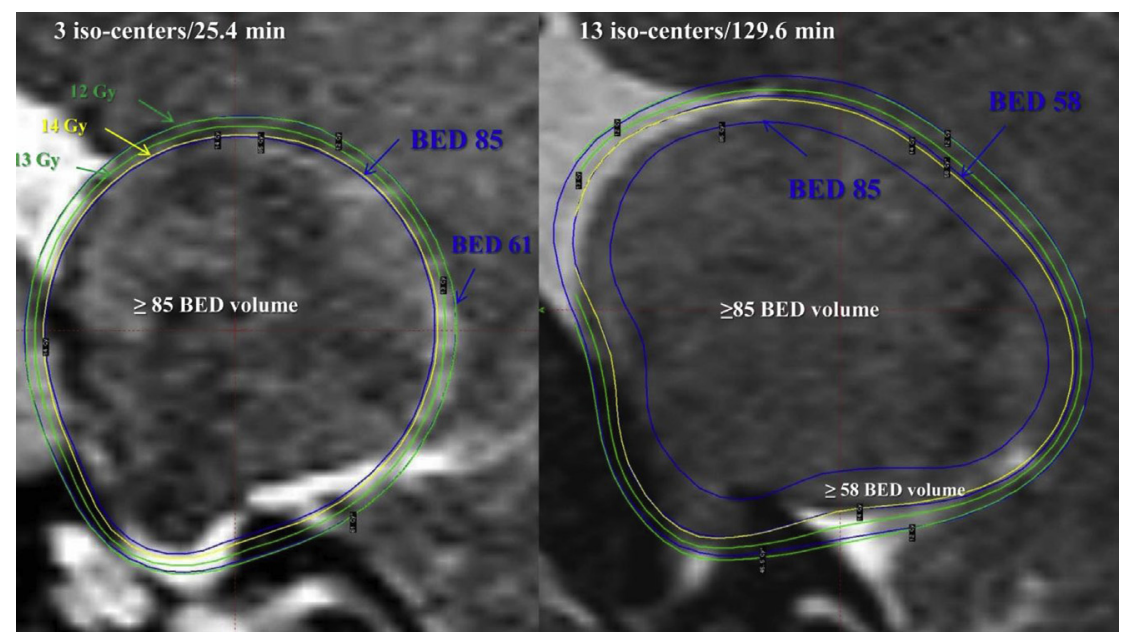

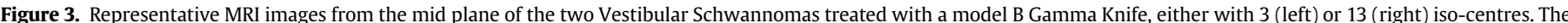

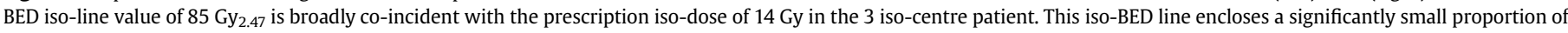

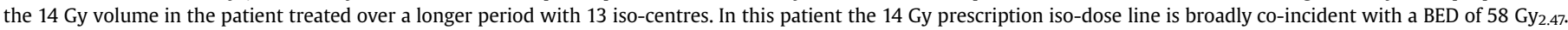


Table 2

Variation in BED values associated with the prescription dose (13 Gy) and the $12 \mathrm{~Gy}$ iso-dose for 3 patients with Vestibular Schwannoma using a Perfexion ${ }^{\circledR}$ Gamma Knife (most representative and the range of values is given).

\begin{tabular}{lll}
\hline Patient no. & Physical dose $(\mathrm{Gy})$ & $\begin{array}{l}\text { Representative BED } \\
\text { (range of BED values) }\left(\mathrm{Gy}_{2.47}\right)\end{array}$ \\
\hline 1 & 13 & $71.5(65.04-72.17)$ \\
& 12 & $61.5(55.96-62.93)$ \\
2 & 13 & $68.5(65.26-69.67)$ \\
3 & 12 & $59.0(56.73-59.53)$ \\
& 13 & $59.5(56.4-61.16)$ \\
& 12 & $52.5(49.14-53.26)$ \\
\hline
\end{tabular}

available radiobiological data for both cells irradiated in vitro [5] and for in vivo animal studies many of which related to the influence of dose protraction on the probability of normal tissue complications [10]. Studies using either continuous radiation exposures, with different dose-rates, or studies involving two or more doses per day with variable incomplete repair intervals between them have been used to obtain information related to the kinetics of repair of sub lethal radiation damage [9], albeit for larger irradiated volumes. These studies have clearly identified a fast component of repair of sublethal radiation injury with a half time of approximately $0.2 \mathrm{~h}$, which infers that repair of sublethal radiation injury is an important factor in RS treatments [15]. Moreover, in a previously unpublished study, that involved the uniform irradiation of a $10 \mathrm{~cm}$ length of the spinal cord of pigs (Hopewell et al., previously unpublished data), a marked difference in response was seen when doses were delivered at two different dose-rates and therefore different overall irradiation times. Accounting for planned gaps in the treatment protocol in this experimental study a total dose of $25 \mathrm{~Gy}$ was delivered in approximately $25 \mathrm{~min}$ or $140 \mathrm{~min}$, for doserates of 0.9 and $0.2 \mathrm{~Gy} / \mathrm{min}$, respectively (for further clarification see text to Fig. 5). For this dose the incidence of late radiation induced myelopathy was reduced from $100 \%$ to $0 \%$ for the short vs

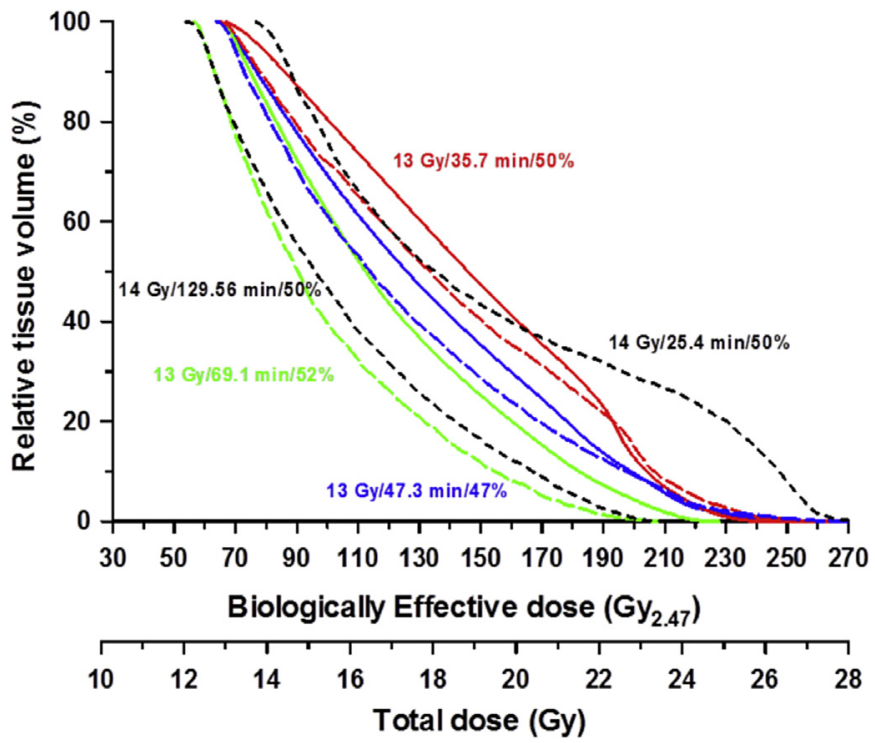

Figure 4. Dose volume histograms (solid lines) for three patients treated in different overall treatment times with a prescription dose of $13 \mathrm{~Gy}$ to between the 47 and $52 \%$ iso-dose using a Perfexion ${ }^{\circledR}$ Gamma Knife. The corresponding BED volume histograms (broken lines) are plotted for comparison along with the two BED volume histograms for the two patients treated with a model B Gamma Knife to a higher prescription isodose of $14 \mathrm{~Gy}$ (black lines). Despite the difference in prescription dose the biological effectiveness off all treatments were in the same range. the long overall treatment times, respectively. Below and above these two doses, delivered using the different dose-rates, the incidence of myelopathy was clearly dose-related (Fig. 5). The $\mathrm{ED}_{50}$ values ( \pm standard error), the doses associated with a $50 \%$ incidence of radiation myelopathy could be calculated from these dose-effect curves; they were highly significantly different. Based on the experimentally derived $\mathrm{ED}_{50}$ value of $21.13 \mathrm{~Gy}$ for the short treatment, an equivalent value of $26.78 \mathrm{~Gy}$ was retrospectively calculated for the long treatment, assuming the same repair parameters used in the present study of Gamma Knife patients. This calculated value of was in good agreement with the experimentally observed value of $27.02 \mathrm{~Gy}$. A physical prescription dose of $25 \mathrm{~Gy}$ is frequently used for the treatment of arterial venous malformations (AVM's) for which overall treatment times, including gaps, can be in excess of the range associated with the pig study i.e. $20-230$ min (P Blackburn, personal communication, 2012) and so this comparison in the pig spinal cord, where gaps between beams similar to those associated with the Model B Gamma Knife, has considerable relevance to the current practice of RS. Even an overall treatment time of 230 min still only represents less than 2 half-times for the repair of sublethal damage via the slow component of $2.16 \mathrm{~h}$ (129.6 min) and thus for all RS treatments the fast component of repair, with a half time for repair of approximately $12 \mathrm{~min}$, is the dominant factor that would be responsible for a loss of efficacy with progressive escalation of the treatment time. A modification of the basic BED equation, to just incorporate a single fast component of repair, was proposed by Joiner et al. [5], in relation to dose protraction in IMRT, when defining the relationship for small (short treatment times) vs large fractions (longer treatment times), for a number of normal tissues represented by biphasic repair kinetics. This methodology would ignore the potentially significant contribution to the BED of the slow repair process i.e. slow repair would not be taken into account and would potentially lead to an incorrect value estimate for the BED.

All of the parameters, use in the present analysis, were experimentally derived from a sufficiently powered study involving only

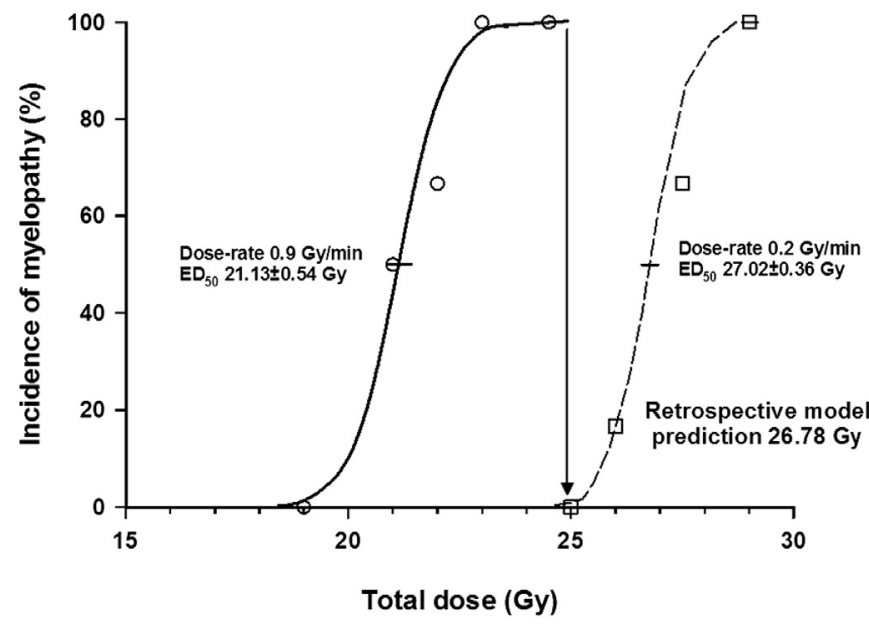

Figure 5. Dose-related changes in the incidence of radiation induced myelopathy in the pig, developing 7-16 weeks after irradiation, as a result of selective necrosis of spinal cord white matter tracks. Irradiation was given at a dose-rate in the spinal cord of either $0.9 \mathrm{~Gy} / \mathrm{min} \mathrm{[16]}$ or later at a dose-rate of $0.2 \mathrm{~Gy} / \mathrm{min}$. In addition to the $\sim 5 \mathrm{~min}$ gaps between opposed fields, in the case of the longer exposures, at the lower doserate, irradiation was stopped for $\sim 5 \mathrm{~min}$ for the treatment to each side to check field positions. The $\mathrm{ED}_{50}(+\mathrm{SE})$, for myelopathy, was calculated from the dose incidence data for each study. For the lower dose-rate study the $\mathrm{ED}_{50}$ was also calculated using the repair parameters for the comparison of RS cases. 
single dose exposures of relatively large target volumes, delivered at a wide range of differing dose rates [10]. Like all experimentally derived parameter they were associated with a level of uncertainty. For example the $95 \%$ confidence interval associated with the $\alpha / \beta$ ratio of $2.47 \mathrm{~Gy}$ was $1.50-3.95 \mathrm{~Gy}$. The use of a different $\alpha / \beta$, within this range, would change the absolute value of the BED values obtained but would not influence the ranking of patients. The effects of changes in the repair parameters has been discussed on theoretic grounds in a previous study [3], where the value of the partition coefficient between the fast and slow component of repair had the greatest influence on the BED value. The effect of changing the size of gaps, between iso-centres, was also considered in relation to the Model B Gamma Knife [3]. BED values declined as the size of the gap increased, i.e. when overall treatment time, was increased. Gaps with Perfexion ${ }^{\circledR}$ are very small, $0.1 \mathrm{~min}$, and constitutes only a small percentage to the overall treatment time. In radiobiological terms they can be considered to be continuous exposures, albeit, that at a given location within the treatment volume the dose-rate varies over the treatment exposure.

In a RS study related to the treatment of trigeminal neuralgia, typically delivered by a single continuous exposure, a BED model with a single half-time was used to compare the biological effectiveness of the treatment with protraction of the dose as the activity of the Cobalt-60 sources declined [12]. One of a number of failings of this study, as discussed previously [3], was that dose-rate was not expressed at the $100 \%$ iso-dose in individual patients but as the machine calibration factor, known as the reference dose-rate, which decreases with the decay of the Cobalt-60 sources. This dose-rate does not reflect the actual dose-rate in the tissues of individual patients and as such has should not be used to infer any differences in biological significance. The other error was the selection of a single long half-time for repair of $6.5 \mathrm{~h}$ for the BED calculations. The origins of this selection was not indicated in the original publication but it would appear that the value came from a study by Landuyt et al. [13] where a long half-time for repair of $6.4 \mathrm{~h}$ was found but in association with a short half-time of $0.25 \mathrm{~h}$. Repair was defined by a composite bi-exponential function and thus half-times cannot be selected as an either/or in isolation but must be used together, a factor that cannot be emphasized enough. In addition, the simple BED model [14] with no repair components added, should not be used in relation to RS treatments as that model only applies to acute exposures that are short compared with the short component of repair and the time between isocentres (or the inter-fraction time) is much greater than the longer repair half-time. Such short exposures, relative to the fast half-time for repair, are not relevant to single session or multisession RS. It should be pointed out that the generalised bi-phasic model effectively reduces to the simple standard LQ model when appropriate conditions, such as those discussed above, are applicable to the irradiation protocol $[7,14]$. Further the $\alpha / \beta$ ratio also has the same value for both formulations; however, it is important that the most appropriate formula is used in any analysis.

The Model B treatment plans, used in the present analysis, represented the maximum range of variation in the protocol. This was both in terms of the least number of iso-centres in the shortest overall treatment time and the most iso-centres used in the longest overall treatment time at the Cromwell Hospital Gamma Knife Centre, London, in the period 1999 to 2005, for a physical prescription dose of $14 \mathrm{~Gy}$. Thus the BED values of 85 and $58 \mathrm{~Gy}_{2.47}$ represent the maximum variation in biological effective dose in patients treated with this method and prescription dose [3]. Since these BED values also represent the highest BED to the normal brain stem, albeit to a small volume, it is interesting to compare these values with the BED values commonly delivered to this region using conventional fractionated radiotherapy. The conventionally quoted tolerance dose of the brain stem and spinal cord is a total dose of 50 Gy given as 25 daily (5 times week) fractions of $2 \mathrm{~Gy}$. Using the same parameters as adopted for the present analysis this is equivalent to a BED of $90.5 \mathrm{~Gy}$ 2.47. A more conservative approach in some radiotherapy centres would be to limit the brain stem dose to $44 \mathrm{~Gy}$ ( 22 fractions of $2 \mathrm{~Gy}$ ) for which the BED is $79.5 \mathrm{~Gy}_{2.47}$. The BED of $85 \mathrm{~Gy}_{2.47}$, obtained for the patient treated in $25.4 \mathrm{~min}$ with three iso-centres is clearly in the range usually accepted as representing brain stem tolerance to conventional radiotherapy. A total dose of $30 \mathrm{~Gy}$ (10 fractions of $3 \mathrm{~Gy}$ ) is also conventionally accepted as representing whole brain tolerance. This treatment is associated with a BED of $66.4 \mathrm{~Gy}_{2.47}$ and thus the second patient treated with a prescription dose of $14 \mathrm{~Gy}$ in 129.6 min (13 iso-centres) received a lower maximum BED to the brain stem than would normally be accepted for the whole brain, even if so called 'hot spots' on the prescription in this patients did exceed a BED of $\sim 65 \mathrm{~Gy}_{2.47}$.

In the present study, the hypothetical maximum BED values would be 93.4 and $81.4 \mathrm{~Gy}_{2.47}$ for 14 and $13 \mathrm{~Gy}$, respectively. Such values are calculated using the basic BED equation [4], which assumes that doses are delivered in a short time relative to the fast half-time for repair and thus that repair of sublethal radiation damage is not a factor in treatments. Such an assumption is clearly inappropriate for either single or multi-session RS treatments [15]. Thus the basic BED equation should not be used for estimating the equivalent dose when converting from experience gained from treatment with single treatment sessions into multiple treatment sessions using BED equivalence.

Based on the not unreasonable assumption for these slowly growing tumours that the repair parameters are similar to those for the normal central nervous system then the tumour in the patient receiving the more complex treatment in $129.6 \mathrm{~min}$ received a lower spectrum of biologically effective doses than the patient receiving the simpler 3 iso-centre treatment. Using a BED value of $85 \mathrm{~Gy}_{2.47}$ as a reference then only $60 \%$ of the tumour volume in the more complex plan received this higher biologically effective dose.

The treatment plans for the three patients treated with the Perfexion ${ }^{\circledR}$ Gamma Knife, received a prescription dose of $13 \mathrm{~Gy}$. These are representative of typical treatments delivered at the Cromwell Hospital Gamma Knife Centre, from 2009 to 2013. Despite the use of a lower prescription dose of $13 \mathrm{~Gy}$ the range of maximum BED values to the normal brain stem were still in the range found for the two patients treatment with the Model B Gamma Knife where the prescription dose was 7.7\% higher (14 Gy). However, it should not be assumed that total physical doses delivered using the Perfexion ${ }^{\circledR}$ Gamma Knife will be consistently biologically higher than those delivered using the Model B Gamma Knife. For example, because of the difference in the relationship between beam on and off times, in association with the overall treatment times, a similar overlap in the range of BED values was also seen in a larger group of patients treated with the Model B Gamma Knife [9]. BED values of between 60 and 70 were most representative of a prescription dose of both $13 \mathrm{~Gy}$, when the overall treatment time varied between $54.55 \mathrm{~min}$ (3 iso-centres) and $124.25 \mathrm{~min}$ ( 8 iso-centres), and $12 \mathrm{~Gy}$, when the overall treatment time varied between 29.5 min ( 2 iso-centres) and 50.47 min (5 iso-centres). This would imply that when overall treatment times vary, as they routinely do in RS, then the concept of treatment planning using physical radiation dose does not necessarily reflect the biological effectiveness of the treatments received, both between patients and even within the same patient along the same iso-dose line.

The current prototype research version of GammaPlan does not allow treatment plans to be conformed to the target using a 
selected prescription BED value. It only allows BED values to be retrospectively calculated for individual voxels. It has been clearly demonstrated that the BED value is a function of the overall treatment time, and the dose/dose-rate protocol, including the number of iso-centres and gaps in the treatment delivery. Although the hypothetical maximum BED value is related to the total physical dose, this is based on the assumption that the total dose is delivered in a short time relative to the fast half-time for repair. Actual plans with differing physical prescription doses were associated with the same BED because of the other variables associated with the different treatment plans. Naturally it would be of interest to generate BED iso-lines to support treatment planning. This requires information about the dose-rate history in every voxel in the calculation matrix to be extracted from GammaPlan $^{\odot}$, as well as the radiobiological parameters (repair times and $\alpha / \beta$ values of tumours and organs at risk) characterizing the relevant BED model. The value of these parameters is well established for CNS tissue and may be equally applicable to some, if not all, brain tumours. Planning according to iso-BED would be similar to planning to a total prescription physical iso-dose with the exception that BED planning would be done with absolute prescription BED values and thus the total physical dose delivery would vary according to the complexity of the plan to adequately cover the target and the time related decay in the activity of the cobalt-60 sources. High conformity to the iso-BED line would then be an objective as well as a steep fall off of BED to spare the normal tissues and organs at risk. Manual BED planning would be feasible for experienced planners but the creation of an optimization tool would ease the creation of complex plans with several conflicting objectives and could be a helpful tool for beginners in treatment planning.

\section{Conclusion}

Significant variations in overall treatment time have a substantial effect on the likely biological effectiveness of a given prescription dose in RS. The biological effectiveness of a given physical prescription dose will decline as the overall treatment time increases, as loss of effectiveness is related to the repair of sublethal radiation-induced damage over more extended periods of exposure. These findings provide important additional information with respect to how the biologically effective dose (BED) varies with the considerable variation in the treatment parameters between different patients. They strongly support the view that overall treatment time is perhaps the most important of the different variables associated with BED treatment planning. This information will help in the design of a comprehensive retrospective studies to compare BED with effect and/or complication probability in radiosurgery.

\section{Acknowledgements}

The authors thank $\mathrm{Mr}$ Alexander Polonsky, Gamma Knife Centre, Cromwell Hospital for sorting out the treatment details of the Perfexion ${ }^{\circledR}$ Gamma Knife cases used in the present analysis. The manuscript is based, in part, on a presentation at the 1st SYRA3 COST Conference, Radiation Therapy with Synchrotron Radiation: Achievements and Challenges, Krakow, Poland (24th to 25th March 2014). The COST Action TD1205 (SYRA3) is thus gratefully acknowledged for its support.

\section{References}

[1] Paddick I. A simple scoring ratio to index the conformity of radiosurgical treatment plans. Technical note. J Neurosurg 2000;93(3 Suppl.):219-22.

[2] Paddick I, Lippitz B. A simple dose gradient measurement tool to complement the conformity index. J Neurosurg 2006;105(3 Suppl.):194-201.

[3] Hopewell JW, Millar WT, Lindquist C. Radiobiological principle: their application to gamma knife therapy, current and future management of brian metastasis. In: Kim DG, Lunsford LD, editors. Prog Neurol Surg, vol. 25; 2012. p. 39-54.

[4] Hopewell JW, Millar WT, Lindquist C, Nordström H, Lidberg P, Gårding J. Application of the concept of biologically effective dose (BED) to patients with vestibular schwannomas treated by radiosurgery. J Radiosurg SBRT 2013;2:259-71.

[5] Bedford JS, Mitchell JB. Dose-rate effects in synchronous mammalian cells in culture. Radiat Res 1973;54:316-27.

[6] Joiner MC, Mogili N, Marples B, Burmeister J. Significant dose can be lost by extended delivery times in IMRT with $\mathrm{x}$ rays but not high-LET radiations. Med Phys 2010;37:2457-65.

[7] Millar WT, Canney PA. Derivation and application of equations describing the effects of fractionated protracted irradiation, based on multiple and incomplete repair processes. Part I. Derivation of equations. Int J Radiat Biol 1993;64:275-91.

[8] Millar WT, Hendry JH, Canney PA. The influence of the number of fractions and bi-exponential repair kinetics on biological equivalence in pulsed brachytherapy. Br J Radiol 1996;69:457-68.

[9] Millar WT, van den Aardweg GJMJ, Hopewell JW, Canney PA. Repair kinetics in pig epidermis: an analysis based on two separate rates of repair. Int J Radiat Biol 1996;69:123-40.

[10] Pop LA, Millar WT, van der Plas M, van der Kogel AJ. Radiation tolerance of rat spinal cord to pulsed dose rate (PDR-) brachytherapy: the impact of differences in temporal dose distribution. Radiother Oncol 2000;55:301-15.

[11] Millar WT, Hopewell JW. Effects of very low dose-rate 90Sr/90Y exposure on the acute moist desquamation response of pigskin: comparison based on predictions from dose fractionation studies at high dose-rate with incomplete repair. Radioth Oncol 2007;83:187-95.

[12] Balamucki CJ, Stieber VW, Ellis TL, Tatter SB, Deguzman AF, McMullen KP, et al. Does dose-rate affect efficacy? The outcomes of 256 gamma knife surgery procedures for trigeminal neuralgia and other types of facial pain as they relate to the half-life of cobalt. J Neurosurg 2006;105:730-5.

[13] Landuyt W, Fowler J, Ruifrok A, Stüben G, van der Kogel A, van der Schueren E. Kinetics of repair in the spinal cord of the rat. Radiother Oncol 1997:45:55-62.

[14] Thames HD. An 'incomplete repair' model for survival after fractionated and continuous irradiations. Int J Radiat Biol 1985;47:319-39.

[15] Sayer FT, Sherman JH, Yen CP, Schlesinger DJ, Kersh R, Sheehan JP. Initial experience with the eXtend System: a relocatable frame system for multiplesession gamma knife radiosurgery. World Neurosurg 2011;75:665-72.

[16] Hopewell JW, van den Aardweg GJMJ, Morris GM, Rezvani M, Robbins MEC, Ross GA, et al. Unsaturated lipids as modulators of radiation damage in normal tissues. In: Horrobin DF, editor. New approaches to cancer therapy: unsaturated lipids and photodynamic therapy. London: Churchill Communications; 1994. p. 88-108. 